\title{
Magnetization dynamics of the ferrimagnet CoGd near the compensation of magnetization and angular momentum
}

\author{
M. Binder, ${ }^{1}$ A. Weber, ${ }^{1}$ O. Mosendz, ${ }^{2}$ G. Woltersdorf, ${ }^{1}$ M. Izquierdo, ${ }^{3}$ I. Neudecker, ${ }^{1}$ J. R. Dahn, ${ }^{4}$ T. D. Hatchard, ${ }^{5}$ \\ J.-U. Thiele, ${ }^{6}$ C. H. Back, ${ }^{1}$ and M. R. Scheinfein ${ }^{2}$ \\ ${ }^{1}$ Department of Physics, Universität Regensburg, 93040 Regensburg, Germany \\ ${ }^{2}$ Department of Physics, Simon Fraser University, Burnaby, British Columbia V5A 1S6, Canada \\ ${ }^{3}$ Synchrotron SOLEIL L'Orme des Merisiers Saint-Aubin-Boîte Postale 48, 91192 Gif-sur-Yvette, France \\ ${ }^{4}$ Department of Physics, Dalhousie University, Halifax, Nova Scotia B3H 3J5, Canada \\ ${ }^{5}$ Department of Chemistry, University of Alberta, Canada \\ ${ }^{6}$ San Jose Research Center, Hitachi Global Storage Technologies, 650 Harry Road, San Jose, California 95120, USA
}

(Received 8 August 2006; revised manuscript received 31 August 2006; published 9 October 2006)

\begin{abstract}
Magnetization dynamics in alloys of ferrimagnetic CoGd have been studied in the vicinity of the magnetization and angular momentum compensation point as a function of alloy composition and temperature. In agreement with standard mean-field treatments of the dynamics of the total magnetization we observe an increase of the precessional frequency and the effective damping parameter near the angular momentum compensation point. We demonstrate the consistency of the magnetization dynamics extracted from frequency domain methods such as ferromagnetic resonance and time resolved laser pump-probe measurements.
\end{abstract}

DOI: 10.1103/PhysRevB.74.134404

PACS number(s): 75.40.Gb, 75.50.Gg, 76.50.+g

Transition metal (TM) rare earth (RE) ferrimagnets are ideal canonical systems to probe magnetization dynamics. ${ }^{1}$ Typically, TM-RE alloys are nearly amorphous materials. The TM sublattice is antiferromagnetically (AF) coupled to the RE sublattice. When the coupling is strong, as, e.g., in CoGd, there are two transition temperatures, the magnetization compensation temperature $T_{M}$ where $M_{\mathrm{Gd}}=M_{\mathrm{Co}}$, and the angular momentum compensation temperature $T_{L}$, where $M_{\mathrm{Gd}} / \gamma_{\mathrm{Gd}}=M_{\mathrm{Co}} / \gamma_{\mathrm{Gd}}$, and $\gamma$ is the gyromagnetic ratio. These temperatures are sensitive functions of the relative concentration. At the magnetic compensation temperature, applied magnetic fields cannot couple to the magnetization to alter its energy since $M_{\mathrm{Gd}}-M_{\mathrm{Co}}=M_{\mathrm{eff}}=0$. Angular momentum is quenched at the angular momentum compensation point, where the AF coupled sublattices gyrate $180^{\circ}$ out of phase about the magnetic field. Studying the dynamics in ferrimagnetic systems are complicated by these tightly coupled AF sublattices. As $T_{L}$ is approached from low temperatures, the phenomenological mean-field damping parameter $\alpha_{\text {eff }}$ which governs how fast the system as a whole dissipates energy increases quickly, and the gyromagnetic frequency changes sign as the angular momentum of the dominant sublattice changes from Gd to Co. An ideal ferrimagnet should dissipate angular momentum instantaneously at $T_{L}{ }^{1,2}$ CoGd was chosen for this study because $T_{M}$ and $T_{L}$ are very close to each other, and the intrinsic orbital moment of Gd is essentially zero, thereby eliminating additional loss channels due to spin-orbit coupling. ${ }^{3}$ We compare experimental results obtained by a frequency domain method used to study the dynamics of the total magnetization of $M_{\text {eff }}$ namely, ferromagnetic resonance (FMR) — to time domain ultrafast laser pump/probe experiments.

The most straight forward method to excite magnetization dynamics uses strong magnetic field pulses that couple directly to the magnetization (spin). ${ }^{4,5}$ These field pulses are typically produced by external sources. However, these methods cannot excite the magnetization at the magnetiza- tion compensation point in a ferrimagnet since there is no net magnetic moment one can couple to. Another method to excite spin-systems employs ultrashort laser pulses that alter the magnetic system by heating across a critical temperature (Curie, Néel, or compensation temperature), ${ }^{6,7}$ by altering the equilibrium direction of the magnetization, ${ }^{8}$ or by driving current directly through photon absorption in a semiconductor underlayer. ${ }^{9}$

In the CoGd samples studied in this paper the laser pulses predominantly excite (and probe) the Co sublattice since the corresponding spin-split bands are close to the Fermi level $\left(E_{F}\right)$. By contrast, the spin-split bands in Gd are spatially localized and lie $\sim 8 \mathrm{eV}$ below $E_{F} \cdot{ }^{10,11}$ In the case of pure optical excitation, the laser pulse rapidly disturbs electrons near $E_{F}$ in the sample. After thermalization, energy is subsequently transferred to the spin and phonon systems leading to a sudden change of the magnitude and/or direction of the internal magnetic field. Apart from ultrafast demagnetization, the modified internal field can cause the magnetization to precess around a new equilibrium position thereby triggering the observed precessional motion of the magnetization. ${ }^{8}$ In this article we focus on the precessional motion of the magnetization and its damping behavior on ps time scales. The unique capability of ultrafast optical pumping allows us to excite the ferrimagnetic system at the compensation points and to explore the dynamics. We observe the expected increase of both the precessional frequency and mean-field effective damping parameter near the compensation points and are able to excite the ferrimagnetic system with the laser pulses in the vicinity of both, the magnetization and angular momentum compensation point. Even when $H_{\mathrm{C}}$ exceeds our maximum external applied field and we are not able to record dc-hysteresis curves, it is still possible to excite and observe magnetization oscillations of the Co sublattice.

At the magnetization compensation point the coercivity becomes large in a ferrimagnet and it is essentially impossible to change the direction of the magnetization. However, 


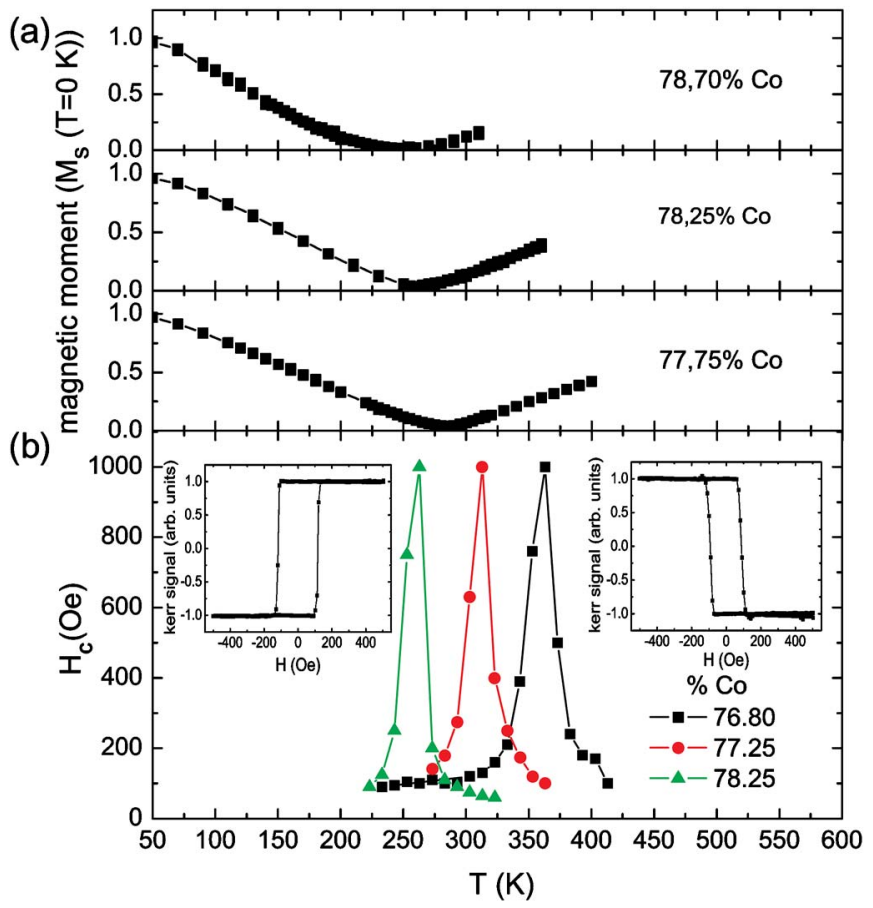

FIG. 1. (Color online) (a) Remanent magnetization as measured by SQUID and (b) coercive field as determined from magneto-optic Kerr effect measurements as a function of CoGd alloy composition and temperature. The left (right) inset shows a hysteresis loop measured below (above) the magnetization compensation point. Note the reversal of the hysteresis loops. All data shown in this figure were obtained from sample set (B).

in a pulsed laser heating experiment the laser predominantly couples to the electrons near $E_{F}$. In the CoGd ferrimagnet the Co moment is mostly carried by the spin-split $3 d$ band near $E_{F}$. The effective moment of Gd close to $E_{F}$ comprises less than $5 \%$ of Gd's total moment which is principally contained in the deep spin-split $4 f$ states. ${ }^{10}$ Note that static hysteresis loops change sign as one passes $T_{M}$ from one side of the magnetization compensation point to the other [see hysteresis loops in Fig. 1(b) below and above the magnetic compensation temperature]. The laser pulse energy is initially deposited into the Co sublattice near $E_{F}$. When the Co sublattice-which is AF exchange coupled to the Gd subsystem by an exchange field well in excess of $50 \mathrm{kOe}-$ is excited, the Gd follows its precessional motion $180^{\circ}$ out of phase. In the specific case when the system is fully compensated, the total saturation magnetization equals zero ( $M_{\text {eff }}$ $=0$ ) irrespective of whether the moments are moving or not. Thus, with a measurement that is sensitive to the total magnetization $M_{\text {eff }}$ it is difficult if not impossible to investigate the dynamics of the independent sublattices near the compensation point. By contrast, in the laser pump-probe experiment, both the excitation and the probe pulses couple predominantly to the transition metal and the persistence of magnetization oscillations of the two coupled sublattices at the magnetization compensation point does not violate the mean-field $M_{\text {eff }}=0$ constraint. Thus, the pump/probe technique can reveal the magnetization dynamics of a system with zero total local magnetic moment.
We use three sets of amorphous CoGd samples. Sample (A) was grown on $\mathrm{Si}(111)$ with native oxide using rf sputtering and provided a continuous concentration gradient across the wafer $(1.43$ percent $/ \mathrm{mm})$ that was confirmed using $\mathrm{X}$-ray analysis. A second gradient sample (B) was grown on $\mathrm{GaAs}(100)$ with a concentration gradient reduced by roughly a factor of 10 to $(0.16$ percent $/ \mathrm{mm})$ in order to better resolve the compensation points. Details of the dc-sputtering procedure for gradient samples have been previously described. ${ }^{12}$ The film thickness was about $3000 \AA$. Samples A and B were capped with transparent $\mathrm{Al}_{2} \mathrm{O}_{3}$ layers to prevent oxidation. A third sample (C) with fixed composition was prepared in order to study the magnetization dynamics as a function of temperature with time-resolved (TR) Kerr-microscopy (MOKE). This sample was deposited by cosputtering from single element targets on a SiN-coated $\mathrm{Si}$ substrate. A $1200 \AA$ thick Au heatsink layer was deposited prior to the deposition of the CoGd film. The film thickness was $300 \AA$, and the composition of the $\mathrm{Co}_{1-x} \mathrm{Gd}_{x}$ films was adjusted to about $x=0.22$ such that $T_{M}$ was just above room temperature.

The static magnetic properties were measured using the magneto-optic Kerr effect, vibrating sample magnetometry (VSM), and by superconducting quantum interference device magnetometry (SQUID). The static properties are summarized in Fig. 1 where we plot the remanent magnetization as a function of temperature and Co concentration [Fig. 1(a)] and the coercive field as a function of temperature and Co concentration [Fig. 1(b)]. In addition typical hysteresis loops below and above $T_{M}$ are shown. We observe the magnetization compensation temperature $T_{M}$ at room temperature at a Co concentration of $77.5 \%$ for sample set (B). In sample sets (A) and (C) the room temperature compensation occurs at slightly different concentrations. For the CoGd alloy the angular momentum compensation temperature $T_{L}$ lies close to $T_{M}$ as the gyromagnetic ratios of $\mathrm{Co}$ and $\mathrm{Gd}$ are almost equal.

For dynamic measurements we focus on samples where $T_{M}$ lies close to room temperature. The dynamic properties in the frequency domain were characterized with FMR at $22 \mathrm{GHz}$ between 250 and $380 \mathrm{~K}$. We also use a vector network analyzer VNA-FMR system described in Ref. 13 which allows us to perform FMR measurements probing only a 90 micron wide stripe of the sample at frequencies between $45 \mathrm{MHz}$ and $20 \mathrm{GHz}$. Figure 2 summarizes the frequency domain data obtained from VNA-FMR and FMR using sample set (B). We plot the effective $g$ factor extracted from the VNA-FMR frequency measured in an external field of $900 \mathrm{Oe}$ as a function of Co concentration (75.5 percent to 80.0 percent) in Fig. 2(a) and observe the characteristic increase of the measured resonance frequency for $\omega_{\text {eff }}$ $=\gamma_{\text {eff }} H_{\text {eff }}$ with $\gamma_{\text {eff }}=g_{\text {eff }}|e| / 2 m_{\mathrm{e}} c$ for $M_{\text {eff }}$ at a Co concentration of $\sim 78 \%$ (similar results were obtained using Brillouin Light Scattering (BLS) but are not shown here). This frequency increase is expected from a mean field model since the equation of motion for the coupled ferrimagnetic system reads $^{1}$

$$
\frac{d}{d t} \vec{M}_{\mathrm{eff}}=-\gamma_{\mathrm{eff}} \vec{M}_{\mathrm{eff}} \times \vec{H}_{\mathrm{eff}}+\frac{\alpha_{\mathrm{eff}}}{M_{\mathrm{eff}}}\left(\vec{M}_{\mathrm{eff}} \times \frac{d}{d t} \vec{M}_{\mathrm{eff}}\right) .
$$




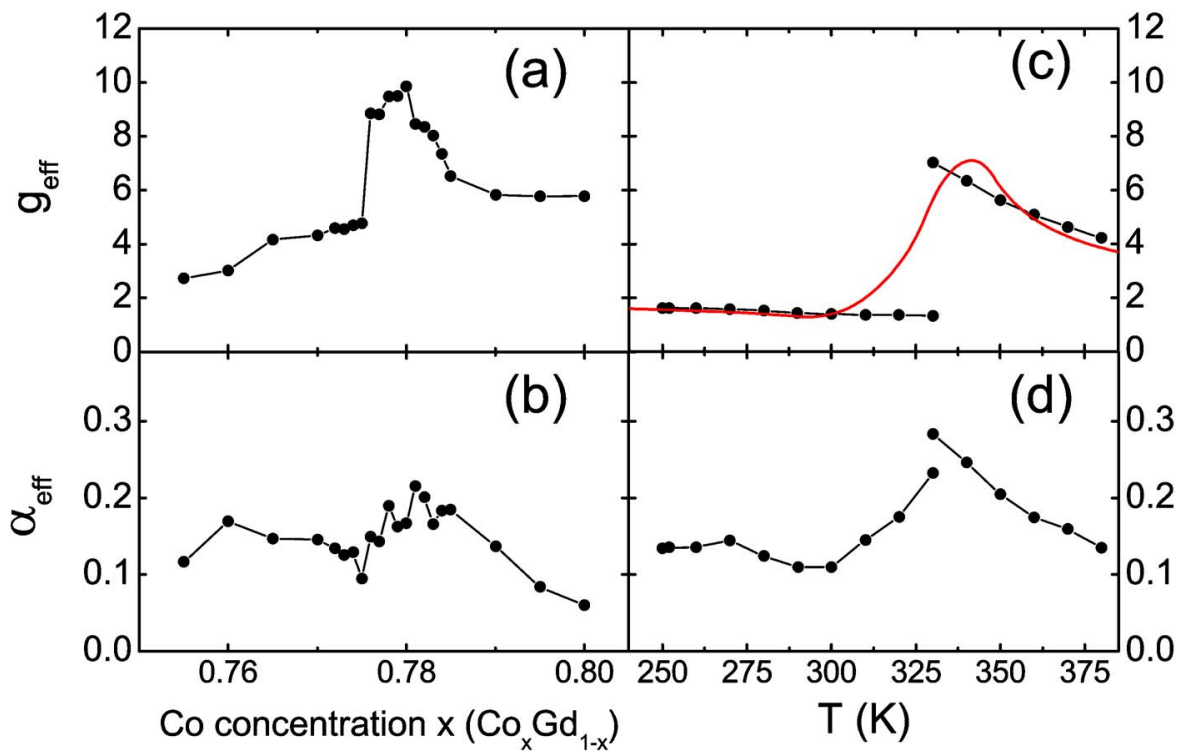

FIG. 2. (Color online) Left: (a) and (b) Data obtained from VNAFMR measurements as a function of CoGd alloy composition. (a) Effective $g$ factor $g_{\text {eff }}$, (b) effective damping parameter $\alpha_{\text {eff. }}$. Measurements were performed at room temperature and in an applied field of $0.9 \mathrm{kOe}$ for VNAFMR. Right: Data extracted from temperature dependent FMR measurements at $22 \mathrm{GHz}$. (c) Effective $g$ factor $g_{\text {eff }}$ vs temperature and (d) effective damping parameter $\alpha_{\text {eff }}$ vs temperature. The red line in (c) corresponds to a fit of $g_{\text {eff }}$ using Eq. (2). All data shown in this figure were obtained from sample set (B).
Here, $\gamma_{\mathrm{eff}}$ is

$$
\gamma_{\mathrm{eff}}(T)=\frac{M_{\mathrm{Co}}(T)-M_{\mathrm{Gd}}(T)}{M_{\mathrm{Co}}(T) / \gamma_{\mathrm{Co}}-M_{\mathrm{Gd}}(T) / \gamma_{\mathrm{Gd}}}
$$

and $\alpha_{\text {eff }}$ is

$$
\alpha_{\mathrm{eff}}(T)=\frac{\alpha_{\mathrm{Co}} \frac{M_{\mathrm{Co}}(T)}{\gamma_{\mathrm{Co}}}+\alpha_{\mathrm{Gd}} \frac{M_{\mathrm{Gd}}(T)}{\gamma_{\mathrm{Gd}}}}{M_{\mathrm{Co}}(T) / \gamma_{\mathrm{Co}}-M_{\mathrm{Gd}}(T) / \gamma_{\mathrm{Gd}}} .
$$

Thus, the ferromagnetic resonance frequency can be described by

$$
\omega_{\text {eff }}(T)=\left|\gamma_{\text {eff }}(T)\right| H_{\text {eff }} .
$$

The exchange resonance frequency is given by ${ }^{14}$

$$
\begin{aligned}
\omega_{\mathrm{ex}}(T) & =\left|\lambda_{\mathrm{ex}}\left[\gamma_{\mathrm{Gd}} M_{\mathrm{Co}}(T)-\gamma_{\mathrm{Co}} M_{\mathrm{Gd}}(T)\right]\right| \\
& =\left|\lambda_{\mathrm{ex}} \gamma_{\mathrm{Co}} \gamma_{\mathrm{Gd}}\left[M_{\mathrm{Co}}(T) / \gamma_{\mathrm{Co}}-M_{\mathrm{Gd}}(T) / \gamma_{\mathrm{Gd}}\right]\right|
\end{aligned}
$$

with the Weiss constant $\lambda_{\text {ex }}$. Close to $T_{\mathrm{M}}$ and $T_{\mathrm{L}}$ the total magnetic system becomes stiffer and the ferromagnetic resonance frequency $\omega_{\text {eff }}$ becomes large. In real samples the singularity in $\gamma_{\text {eff }}$ is bounded due to sample inhomogeneity (RE concentration) and temperature gradients as the temperature approaches $T_{L}$. Furthermore, the exchange mode frequency is expected to tend to zero. In Fig. 2(b) we plot the measured $\alpha_{\text {eff }}$ which also increases around the angular momentum compensation point. When we convert the linewidth to the damping parameter according to $\alpha_{\text {eff }}=\Delta f / f$ we observe a

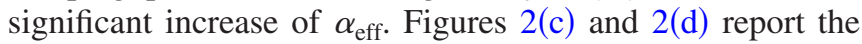
results obtained from FMR measurements as a function of temperature. We show the extracted effective $g$ factor $g_{\text {eff }}$ and the effective damping parameter $\alpha_{\text {eff }}$. Again a clear signature of the (quasi) divergence of $g_{\text {eff }}$ and the increase of $\alpha_{\text {eff }}$ in the vicinity of the compensation points can be observed. The red line in Fig. 2(c) corresponds to a fit using Eq. (2). Based on the SQUID measurements [see Fig. 1(a)] we chose a linear decreasing magnetization for both sublattices with increasing temperature $T$ for the fit. The saturation magnetization at $T=0 \mathrm{~K}$ and the $g$ factor are $M_{s}^{\text {Co }}$ $=1400 \mathrm{emu} / \mathrm{cm}^{3}$ (Ref. 15) and $g_{\mathrm{Co}}=2.18$ (Ref. 16) for Co and $M_{s}^{\mathrm{Gd}}=2060 \mathrm{emu} / \mathrm{cm}^{3}$ (Ref. 15) and $g_{\mathrm{Gd}}=2$ (Ref. 17) for $\mathrm{Gd}$, respectively. Due to the concentration gradient present in the sample the magnetization can never be completely compensated in the FMR measurements. For sample (B) the concentration gradient leads to a spread of RE concentration of about $0.2 \%$ and corresponds to a spread of $T_{L}$ of about $20 \mathrm{~K}$ according to Hansen et al. ${ }^{18}$ This effect was taken into account for the fit. We find a separation of $T_{M}$ and $T_{L}$ of about 30 K. As can be seen in Fig. 2(c) the fit reproduces the FMR
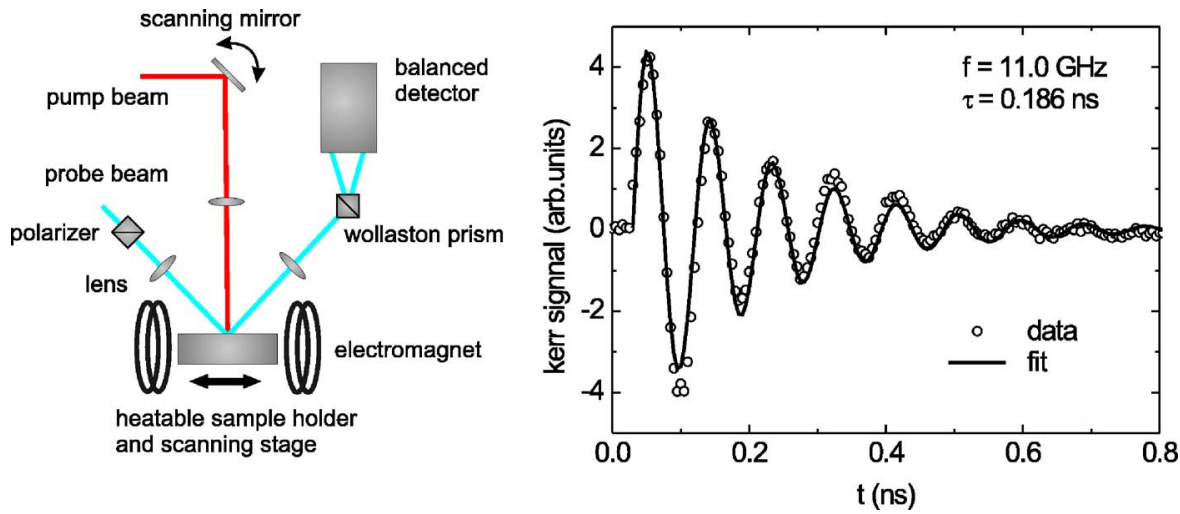

FIG. 3. (Color online) Experimental setup used for the time resolved Kerr effect measurements. The right panel shows time domain data obtained with this setup using sample (A) above the compensation point at an external inplane bias field of 1000 Oe. The solid line is a fit to the data using an exponentially decaying sine function. 
measurements reasonably well. Assuming the same parameters and a typical value of $1 \times 10^{4}$ for $\lambda_{\text {ex }}{ }^{14} \mathrm{Eq}$. (5) predicts the optical exchange mode with a minimum frequency of $980 \mathrm{MHz}$ around the angular momentum compensation temperature $T_{L}$.

In the laser pump/probe experiment, we use a $840 \mathrm{~nm}$ $(1.48 \mathrm{eV})$ laser pulse with $170 \mathrm{fs}$ duration at $260 \mathrm{kHz}$ repetition rate delivered by an amplified Ti:Sapphire laser system to trigger the precession of the magnetization in an externally applied field. The heating pulse is focused into a $50 \mu \mathrm{m}$ diameter spot and a pump fluence of $15 \mathrm{~mJ} / \mathrm{cm}^{2}$ is used. The precession of the magnetization is monitored using the polar Kerr effect with a frequency doubled laser pulse from the same laser system at $420 \mathrm{~nm}(2.95 \mathrm{eV})$ focused into a beam diameter of $15 \mu \mathrm{m}$ resulting in a low fluence of $1.5 \mathrm{~mJ} / \mathrm{cm}^{2}$, as illustrated in Fig. 3. Pump and probe beams are delayed with respect to each other by using an optical delay line providing subpicosecond temporal resolution. Typical time domain data obtained with this setup are shown in the right panel of Fig. 3.

In these experiments the pump laser power is predominantly absorbed by the Co sublattice leading to changes in the equilibrium magnetization causing it to precess [see Figs. 3 and 4]. As the two sublattices are strongly AF coupled, we expect the Gd sublattice to precess $180^{\circ}$ out-of-phase with respect to the Co sublattice. The probe pulse also predominantly samples the Co sublattice. This is substantiated by the fact that when measuring static hysteresis loops as a function of temperature or sample composition, the loops flip when crossing the magnetization compensation point indicating when the majority (larger $M$ ) sublattice is aligned with an externally applied field [see inset Fig. 1(b)]. We also observe a phase shift in the precession of $180^{\circ}$ when crossing the angular momentum compensation point from low temperature [see inset in Fig. 4(a)]. This is expected since the dominant sublattice determines the sense of the gyromagnetic rotation and at the compensation temperature it changes from Gd to Co. To further investigate the magnetization dynamics in the vicinity of the compensation points we now focus on a sample (C) with constant composition of $78 \%$ Co. From VSM measurements we expect $T_{M}$ to be around $390 \mathrm{~K}$ in this sample.

This is confirmed by the time-resolved pump/probe experiments, see the large frequency increase in the vicinity of the compensation temperature in Fig. 4(a). The decay time $\tau$ decreases by about 50\%, see Fig. 4(b). The corresponding increase in $\alpha_{\text {eff }}$ is shown in Fig. 4(d) using the relation $\tau=2 /\left[\gamma_{\mathrm{eff}} \alpha_{\mathrm{eff}}\left(M_{S}+2 H\right)\right]$. Note that the temperature increase by the laser heating (approximately $50 \mathrm{~K}$ ) is included in the temperature presented here. The peak of the damping parameter at the angular momentum compensation point is more pronounced in the data obtained using sample set (A), see Fig. 4(e). In this case TR-MOKE was used to measure $\alpha_{\text {eff }}$ as a function of the Co concentration at room temperature.

The loss mechanisms are regulated by the Gilbert damping constant in the Landau-Lifshitz-Gilbert equation. In the two sublattice mean-field model adopted to characterize a ferrimagnetic system, the effective damping parameter $\alpha_{\text {eff }}$ becomes large at the angular momentum compensation point $T_{L}$.

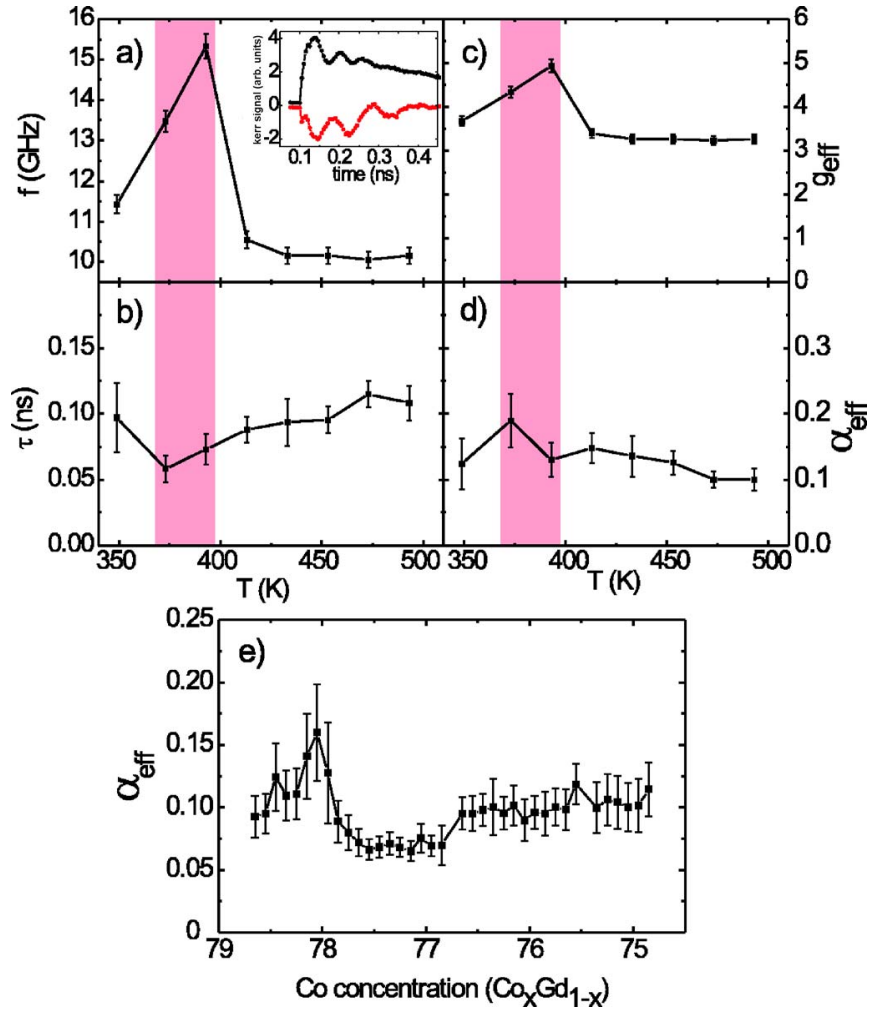

FIG. 4. (Color online) Summary of the time resolved pump/ probe data. In (a) and (b) we report the measured frequency and decay time of the oscillations of the magnetization as a function of temperature for sample $(\mathrm{C})\left(\mathrm{Co}_{78} \mathrm{Gd}_{22}\right)$. The measurements are carried out in a field of 2200 Oe applied at $30^{\circ}$ with respect to the film normal. Again, the data is extracted from traces similar to those shown in the inset. In the inset the black (upper) trace corresponds to data taken just below the angular momentum compensation point, while the red (lower) data corresponds to data taken slightly above the angular momentum compensation point. Notice the $180^{\circ}$ phase shift in the precession of the magnetization. The error bars are obtained by the scatter of the fit results when using different methods to remove the background. The red horizontal bars indicate the position of the compensation region as determined from the divergence of the coercive field in static hysteresis loops and uncertainties in the temperature of the sample. (c) and (d) show the corresponding effective $g$ factor $g_{\text {eff }}$ and damping constant $\alpha_{\text {eff. }}$ (e) shows the dependence of $\alpha_{\text {eff }}$ vs Co concentration for sample (A).

Since the mean-field model contains no explicit changes that modify how each individual sublattice's angular momentum might couple into or out of the system ${ }^{19}$ one can expect the intrinsic (spin-orbit dominated) damping rates to remain essentially constant. ${ }^{2}$ The increased frequency and damping constant are consistent with mean field models. Regardless how the magnetic film was excited, by an external magnetic field (FMR) or an optical laser pulse (TR-MOKE), similar results are obtained. The temperature dependence of the TRMOKE results by Stanciu et al. ${ }^{20}$ agree with our findings. We also find the increase of the precessional frequency $f$ and of the damping parameter $\alpha_{\text {eff }}$ at the angular compensation point $T_{\mathrm{L}}$. However, in contrast to their results we do not observe any evidence of a high frequency optical exchange mode. Far from the compensation region the optical ex- 
change mode is in the range of several THz. The mean field model Eq. (5) predicts that the frequency of the exchange mode drops to zero at the angular momentum compensation point. As a consequence of temperature and concentration gradients (laterally as well as across the film thickness) the divergence of the resonance frequency around the angular momentum compensation point is always limited (see Figs. 2 and 4). Based on the mean field model which leads to the fit shown in Fig. 2(c) we expect that the exchange mode frequency does not drop below several hundred $\mathrm{GHz}$ at $T_{L}$ in our samples. It seems unlikely that in our pump probe TRMOKE experiments the optical mode can be excited coherently. This is probably the reason why we never observed the exchange mode.It may be possible to detect this mode in BLS since there the thermal excitation is measured and coherence is not required.

In conclusion, we have analyzed the damping behavior of a model ferrimagnetic system in the vicinity of the magneti- zation and angular momentum compensation point. We have shown that by using complementary techniques it is possible to study the damping behavior of the total system and of the Co sublattice in detail. We observe a large increase in precessional frequency and damping for the total magnetization in the frequency and the time domain. The time domain study shows that an all optical control of the magnetization dynamics in the vicinity of the compensation point may be feasible. Ferrimagnets close to the compensation points are therefore well suited for high speed manipulation of the magnetization and may be of interest to magneto-optic recording applications.

Financial support by the Deutsche Forschungsgemeinschaft through the Priority Programme 1133 "Ultra Fast Magnetization Processes" is acknowledged. J.R.D. and T.D.H. acknowledge funding from the Canadian Institute of Advanced Research-Nanoelectronics Program.
${ }^{1}$ R. K. Wangsness, Phys. Rev. 91, 1085 (1953).

${ }^{2}$ V. Kambersky, Can. J. Phys. 48, 2906 (1970).

${ }^{3}$ S. G. Reidy, L. Cheng, and W. E. Bailey, Appl. Phys. Lett. 82, 1254 (2003).

${ }^{4}$ W. K. Hiebert, A. Stankiewicz, and M. R. Freeman, Phys. Rev. Lett. 79, 1134 (1997).

${ }^{5}$ C. H. Back, D. Weller, J. Heidmann, D. Mauri, D. Guarisco, E. L. Garwin, and H. C. Siegmann, Phys. Rev. Lett. 81, 3251 (1998).

${ }^{6}$ E. Beaurepaire, J.-C. Merle, A. Daunois, and J.-Y. Bigot, Phys. Rev. Lett. 76, 4250 (1996).

${ }^{7}$ J. Hohlfeld, Th. Gerrits, M. Bilderbeek, Th. Rasing, H. Awano, and N. Ohta, Phys. Rev. B 65, 012413 (2001).

${ }^{8}$ M. van Kampen, C. Jozsa, J. T. Kohlhepp, P. LeClair, L. Lagae, W. J. M. de Jonge, and B. Koopmans, Phys. Rev. Lett. 88, 227201 (2002).

${ }^{9}$ Y. Acremann, M. Buess, C. H. Back, M. Dumm, G. Bayreuther, and D. Pescia, Nature (London) 414, 51 (2001).

${ }^{10}$ S. S. Jaswal, D. J. Sellmyer, M. Engelhardt, Z. Zhao, A. J. Arko, and K. Xie, Phys. Rev. B 35, 996 (1987).

${ }^{11}$ A. Kowalczyk, G. Chelkowska, and A. Szajek, Solid State Commun. 120, 407 (2001).
${ }^{12}$ J. R. Dahn, S. Trussler, T. D. Hatchard, A. Bonakdarpour, J. R. Mueller-Neuhaus, K. C. Hewitt, and M. D. Fleischauer, Chem. Mater. 14, 3519 (2002).

${ }^{13}$ I. Neudecker, G. Woltersdorf, B. Heinrich, G. Gubbiotti, and C. H. Back, J. Magn. Magn. Mater. 307, 148 (2006).

${ }^{14}$ J. Kaplan and C. Kittel, J. Chem. Phys. 21, 760 (1953).

${ }^{15}$ C. Kittel, Introduction to Solid State Physics, (John Wiley \& Sons, New York, 1996).

${ }^{16}$ J. Pelzl, R. Meckenstock, D. Spodding, F. Schreiber, J. Pflaum, and Z. Frait, J. Phys.: Condens. Matter 15, 451 (2003).

${ }^{17}$ Ferromagnetic Materials, edited by E. P. Wohlfahrt (North Holland, Amsterdam, 1980), Vol. 1.

${ }^{18}$ P. Hansen, C. Clausen, G. Much, M. Rosenkranz, and K. Witter, J. Appl. Phys. 66, 2 (1989).

${ }^{19}$ This is only the case if no new pathways for spin-orbit coupling or defect scattering are introduced. To minimize spin-orbit coupling we selected Gd as the rare-earth with $L_{\text {orbit }} \sim 0$.

${ }^{20}$ C. D. Stanciu, A. V. Kimel, F. Hansteen, A. Tsukamoto, A. Itoh, A. Kiriliyuk, and Th. Rasing, Phys. Rev. B 73, 220402(R) (2006). 